\title{
Guided Reflectometry Imaging Unit using Millimeter Wave FMCW Radars
}

\author{
M. Pan, A. Chopard, F. Fauquet, P. Mounaix*, J.-P. Guillet*
}

\begin{abstract}
Frequency Modulated Continuous Wave (FMCW) radar systems in the millimeter and sub-millimeter range are technologically mature for many applicative fields such as automotive and aerospace industries for imaging and non destructive testing. This work reports on a new implementation of a guided FMCW radar reflectometry unit for sensing and imaging applications. Only a terahertz dielectric waveguide is used for signal transmission between the transceiver module and the sample, thus drastically simplifying the experimental setup. Compared to continuous wave guided systems, one of the main advantages granted by the use of FMCW radars in combination with waveguides, is the differentiation capability between the reflected signals generated along the wave guide as parasitic signals or at its probing end as sensing information and therefore improving the expected signal-to-noise ratio. This innovative approach is demonstrated by using a dielectric hollowcore waveguide integrated with two different radar transceivers; the high-performance, III-V based $100 \mathrm{GHz}$ SynView unit as a reference system and a compact, low-cost, PCB-Integrated, 122 GHz transceiver developed by Silicon-Radar GmbH. Both 3D electromagnetic simulations and raster scans are performed to investigate quantitatively the propagation behaviors including the coupling capabilities, dynamic range limitations, beam profile and induced artefacts of the guided FMCW reflectometry system. The feasibility of a simplified guided terahertz FMCW reflectometry probing unit is proven. The integration of a solid immersion lens at the end of the waveguide is also demonstrated for imaging resolution improvement.
\end{abstract}

Index Terms-Guided waves, FMCW Radar, Imaging, Terahertz

\section{INTRODUCTION}

$\mathbf{T}$ HE scaling down of Si-based transistors and the investigations into III-V technologies further push up the operating frequency of solid-state devices towards the terahertz regime [1]. On this basis, high-speed electronic devices with low power consumption and better compactness have been developed in the $100-300 \mathrm{GHz}$ range. Being cost-effective systems supported by continuously improved fabrication techniques, solid-state terahertz devices play an increasingly important role in academic researches and industrial applications [2]. Compared to conventional continuous wave systems combined with 3D imaging reconstruction approaches, such as Shape From Focus [3] or Computed Tomography [4], terahertz and/or millimeter wave Frequency-Modulated ContinuousWave (FMCW) radar systems can natively provide additional

Manuscript received April 09, 2020. The first two authors contributed equally to this work. Asterisk indicates corresponding authors.

M.Pan, A. Chopard, F. Fauquet, P. Mounaix and J.-P. Guillet are with the IMS Laboratory, UMR CNRS 3218, University of Bordeaux, 351 Cours de la Libération 33405 Talence Cedex, FRANCE

A. Chopard is also with Lytid SAS, 8 rue la Fontaine, 92120 Montrouge.

E-mail :patrick.mounaix@u-bordeaux.fr; jean-paul.guillet@u-bordeaux.fr phase information for further result analysis and simplified $3 \mathrm{D}$ reconstructions with suitable resolution, while allowing further processing for improvement [5]. In particular, exploiting a quasi-optical coupling method (using lenses or parabolic mirror) with FMCW systems allows in-depth measurements for non-destructive testing purposes. This technique combines the high sensitivity of FMCW methods with the penetration capabilities of millimeter waves. Based on those benefits, wideband FMCW radars have found suitable application fields in the automotive and aerospace industries [6], [7] and artpainting analysis [8] amongst others for their non-destructive testing capabilities. However, the use of optical components involves tedious alignment and imposes mechanical restrictions along the propagation path. The use of such optical coupling methods limits the development of compact, portable and easily-implementable terahertz measurement systems to a broader scope of applications.

To address this issue, a terahertz waveguide [9] is proposed as an alternative solution. The guided reflectometry concept has already been investigated with some continuous wave sources or pulsed sources [10] in conjunction with different waveguide conceptions. A variety of geometries have been assessed, from a rectangular waveguide coupled to a vector network analyzer at low frequencies for burn damage detection [11], to higher frequencies tests with low-cost Teflon waveguides for remote chemical detection [12] or metallic waveguide for remote endoscopic measurements [13], [14]. Those studies demonstrated the feasibility and the potential of $\mathrm{THz}$ guided reflectometry systems. However, the complexity induced by the optical coupling setups (beam splitter, lenses, parabolic mirrors) remains and limits the progress towards compact guided sensing units.

In our work, the guided reflectometry configuration is highly simplified by testing compact FMCW transceivers with a dielectric thin-wall hollow-core waveguide of suitable dimensions. An optic-free transmission channel between the transceiver and the sample is then ensured with all the benefits of the FMCW radar sensing technique. In the following sections, two guided reflectometry configurations consisting of different compact FMCW radar units are introduced in order to demonstrate adequacy and universality of this approach regarding the employed technology and the front-end of the system. Investigations into the propagation behaviors in the waveguide and final system performances are given, demonstrated and further improved with the integration of an extra termination hemispherical lens. 


\section{GUIDED FMCW RADAR REFLECTOMETRY SYSTEM}

\section{A. FMCW Radar: basic principle}

A simplified architecture diagram depicts, in Fig. 1a, the typical implementation of a monostatic radar transceiver operating in reflection mode. Driven by a cyclic command signal, a linear FMCW reference signal is generated at low frequency by a Voltage Control Oscillator (VCO) or a Phase Locked Loop (PLL) oscillator, which is then fed into the frequency multiplication chain, for signal up-conversion, to reach the desired operating frequency band. A 3-ports coupler leads this probing signal towards the emission antenna while redirecting the reflected signal towards the mixing unit for down-conversion and sampling. Similar bi-static architectures are also practicable but require a partition between the emitting chain and receiving unit, complicating the system and impacting its compactness.

As showed in Fig. 1b, the cyclic signal reflected from the target is delayed with respect to the reference emission sweep and thus gives rise to a beating frequency $f_{b}$ at the mixer's output proportional to the propagation length. Hence the object's distance can be expressed as Eq. 1

$$
\begin{gathered}
d=\frac{c_{0} \Delta_{t}}{2 n}=\frac{c_{0} f_{b}}{2 n} \cdot \frac{T_{s}}{B}, \\
\delta_{\text {res }}=\frac{c_{0}}{2 n B} .
\end{gathered}
$$

where $\Delta_{t}$ is the propagation-induced time delay, $n$ represents the optical refractive index of the propagation media, $f_{b}$ donates the mixer's output beating frequency, $T_{s}$ is the period of a sweep cycle, and B is the sweep frequency bandwidth.

When multiple targets are involved, each object contributes as a given distance-related beating frequency $f_{b_{i}}$, allowing for differentiation and remote sensing capability through data processing steps. Nevertheless, derived from Eq. 1, the longitudinal resolution, $\delta_{r e s}$, is directly correlated to the finite bandwidth (see Eq. 2).

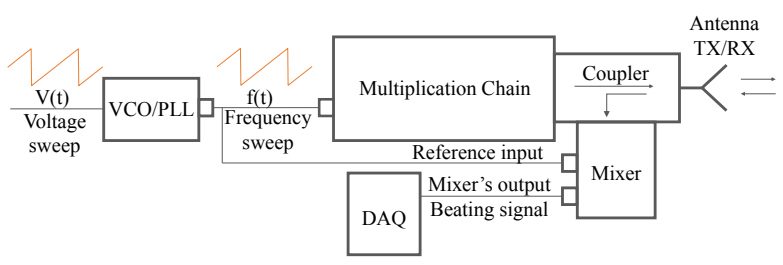

(a)

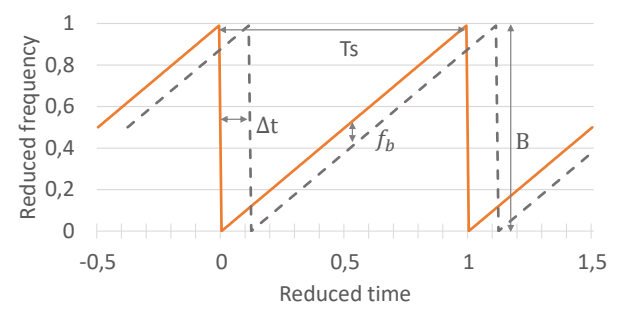

(b)

Fig. 1: (a) Simplified architecture of a monostatic FMCW radar transceiver. (b) FMCW mode of operation: emitted linear frequency sweep reference (solid line) and delayed reflected signal (dashed line) from a distant single object.

\section{1) Si-Based technologies}

From the FMCW mode of operation, the technological implementation for radar transceiver units has been widely investigated through two distinct technologies derived either from silicon-based integrated circuit or from III/V semiconductor diode-based components. Thanks to the continuous improvement of the Si-based transistors processing technology, a steady decrease have been witnessed in those devices production prices. Nowadays, Si-based FMCW radar transceivers are drawing attention in industrial fields thanks to their high integration level, low-cost and versatility. As an example, based on previous generations at $24 \mathrm{GHz}$, radar designs for automotive applications [15] have been developed in the 77 $\mathrm{GHz}$ band with simple bi-static architectures. With a similar design, a $122 \mathrm{GHz}$ transceiver based on SiGe technology [16], [17] is developed by Silicon-Radar GmbH. Apart from the 245 $\mathrm{GHz}$ radar unit under development [18], this transceiver is the only commercially available Si-based unit above $100 \mathrm{GHz}$ on the market to our knowledge. It offers a $6 \mathrm{GHz}$ bandwidth through a bi-static geometry based on separated $2 \times 2$ patch array antennas as RX and TX, on an $8 \times 8 \mathrm{~mm}^{2}$ QFN (Quad Flat No-leads) package.

In our work, this $120 \mathrm{GHz}$ Si-based radar transceiver chip has been integrated amongst the guided radar reflected measurement unit as a low-cost implementation solution. In order to push the investigations on the guided reflectometry unit design, and more especially the coupling capabilities of the radar units with the waveguide, preliminary electromagnetic simulations of the radar transceivers are conducted. The resulting simulated radiation pattern of the Silicon Radar unit, depicts, as expected, a broad far-field emission profile in nearly a half space (a maximal directivity of $11 \mathrm{dBi}$ with an angular width around $47^{\circ}$ and $48^{\circ}$ in E and H-plane, respectively), corresponding to the typical response of such patch array antennas design.

\section{2) III/V-Based technologies}

Compared to Si-based semiconductor technologies, III-V based transistors show advantages in terms of electron mobility, thermal conductivity and operating voltage. Their maximum frequencies have exceeded $1 \mathrm{THz}$, making it possible to realize highly integrated, powerful, complex transmitters and receivers at higher terahertz frequencies. Thanks to the Schottky diodes implementation, $2 \mathrm{THz}$ frequency multiplication chains are achievable [19]. However, III/V-based fully integrated MMIC [20] still displays high frequencies implementation limitations. Hybrid integration is suggested as a compromise solution for high-end applications. It is implemented through the fine tuning of a succession of components in discrete waveguide blocks [6], [21]. III-V based radars have been demonstrated around $100 \mathrm{GHz}, 300 \mathrm{GHz}$ and $600 \mathrm{GHz}$ for different application cases [22], and solutions up to 850 $\mathrm{GHz}$ have been developed by SynView GmbH.

A $100 \mathrm{GHz}$ SynView FMCW transceiver, providing 50 $\mathrm{dB}$ measurement dynamic range, is as well implemented in our guided terahertz FMCW reflectometry unit as a reference system. A mono-static block-integrated architecture in combination with a conical horn antenna ensures the functionality of 
this III-V based transceiver module. For this horn antenna geometry, the conduction of similar characterization simulations depicts a much more suitable emission profile with maximum directivity of $20 \mathrm{dBi}$, while $14.4^{\circ}$ angular width is achieved in the H-plane, and $24.1^{\circ}$ is obtained in E-plane. Particularly, such emission profile allows a increased forward and back signal coupling between the antenna and the waveguide thanks to the improved directivity.

\section{B. Waveguide for remote sensing}

Lying between the microwaves and infrared regions, the development of terahertz waveguides benefits from the advanced technologies in those two domains [9]. However, the absorption loss in dielectrics and skin effect power losses induced by metals in the terahertz band limit the performances of teraherz waveguides. Considering that dry-air is the most transparent terahertz medium, air-core waveguides with different cladding structures (simple hollow-core waveguides [23], [24], metalcoated dielectric waveguides [25] and photonic crystal hollowcore waveguides [26]), have drawn significant attention. Continuous effort is being brought to improve the transmission efficiency through new designs and materials [27], [28]. In this work, a thin-wall hollow-core Polypropylene waveguide is selected to be implemented due to its simple structure, low-loss propagation properties of the cladding material and availability in the commercial market. This type of waveguide exploits the anti-resonant reflections as guiding mechanism [29]. Since the high-index cladding layer acts as a Fabry-Pérot etalon, the air core allows terahertz waves to propagate inside at non-characteristic frequencies. The loose confinement in the air core gives rise to a low-loss propagation. The cladding's characteristic frequencies [23] are defined by:

$$
f_{m}=\frac{m c}{2 t_{c l} \sqrt{n_{c l}^{2}-1}},
$$

where $c$ is the speed of the light in vacuum, $m$ is an integer, $t_{c l}$ and $n_{c l}$ are the thickness and refractive index of the cladding respectively.

In addition to the suitability of the cladding thickness to ensure the guiding mechanism, one of the key parameters to be determined for the selection of such a waveguide, remains its diameter to establish the optimum power coupling between the antennas and the waveguide.

Due to the complexity of the involved anti-resonant guiding mechanism and the irregularities in the emission profiles of the transceivers, electromagnetic simulations have been conducted on the two considered radar units to investigate the impact of the waveguide diameter and positioning on the coupling capabilities. Similarly to the power coupling with the horn antenna geometry, and with the same evolution tendency, the coupling efficiency displays an important dependency on the waveguide diameter for the RX and TX patch antennas geometry of the Silicon-radar unit, (see Fig. 2) and displays a power coupling of $30 \%$ for an optimum $2 \mathrm{~mm}$ waveguide radius.

Experimentally, a $3 \mathrm{~mm}$ radius plastic pipe waveguide with a cladding thickness of $0.158 \mathrm{~mm}$ is chosen thanks to the market availability and ensures a trade-off between coupling

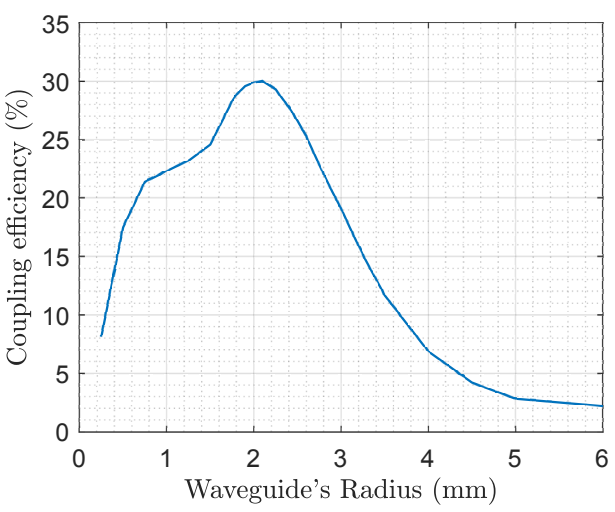

Fig. 2: Evolution of the simulated forward power coupling efficiency on a $300 \mathrm{~mm}$ long guided propagation with respect to the waveguide's diameter for the Silicon radar unit frond-end design.

efficiency and expected optical lateral resolution. According to Eq. 3, with $\mathrm{t}=158 \mu \mathrm{m}$ and $\mathrm{n}=1.49$ (THz-TDS timedomain spectroscopy extracted refractive index), characteristic resonant frequencies of this plastic cladding are expected to be around $860 \mathrm{GHz}$ and $1.72 \mathrm{THz}$ [10], allowing the waveguide to support $100 \mathrm{GHz}$ waves' propagation without significant impacts on the considered FMCW radars bandwidth. Those characteristic non-supported propagation frequencies have been witnessed through a TDS investigation on the same waveguide, demonstrating losses peaks around $840 \mathrm{GHz}$ and 1.7 THz.

\section{Experimental setup}

As a simplified guided configuration, an optics-free single communication path between the FMCW module and sample is provided by the previously mentioned hollow-core dielectric waveguide. In order to ensure an adequate coupling, the waveguide is properly placed with respect to the transceiver. No dedicated quasi-optical setup or beam shaping elements are employed. As a simple optimization, a foam support, acting as terahertz absorber, is placed nearby the coupling area to neutralize the reflected parasitic signals induced by the noncoupled echoes, resulting in a significant signal improvement.

Unlike from guided continuous wave reflectometry measurement techniques, where the detected signal is a superposition of all the contributions that are generated along the waveguide, the phase information provided by guided FMCW techniques unlocks depth sensing capabilities and allows a differentiation of those contributions. More specifically, beside a sensing capability along the waveguide with distance differentiation, it ensures a drastic improvement of the signalto-noise ratio thanks to the selection of the sensing distance, linked to the desired contributions, while not taking into account any parasitic signal. This capability represents the main motivation behind the implementation of waveguides with millimeter wave FMCW radar units rather than continuous wave sources, even though, the low longitudinal resolution of the considered radar units does not allow depth sensing measurements at the waveguide's output.

With a purpose of demonstrating the simplicity of this approach regardless of the operated technology, system emission 
frond, packaging, and price, the FMCW guided reflectometry unit is established with two specifically distinct transceivers.

Implementing the $100 \mathrm{GHz}$ SynView radar head as a reference configuration (see Fig. 3a), the waveguide is directly inserted in the horn antenna to maximize the forward-coupled power while ensuring a proper centering. This symmetric positioning of the waveguide and the linearly- polarized emission profile of horn antenna are beneficial parameters to excite the air-core propagation modes for low-loss transmission along the waveguide.

As a compact and low-cost approach, the $122 \mathrm{GHz}$ SiliconRadar chip displays a full integration with two separated patch array antennas as TX and RX. Although the coupling processes are no longer symmetrical due to a $3 \mathrm{~mm}$ TX-RX separation, the positioning of the hollow-core waveguide is centered on the focal point as defined in [16], equidistant from the TX and RX patch antenna arrays, while the foam absorber is still employed to suppress parasitic non-coupled signals (see Fig. 3b).

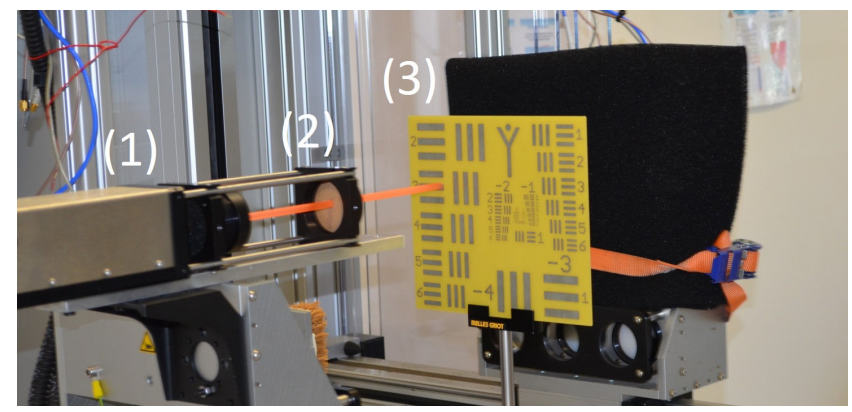

(a)

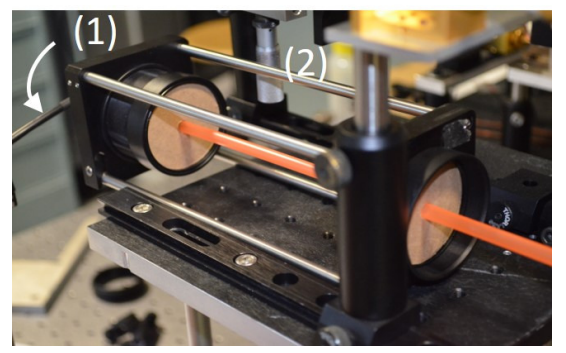

(b)

Fig. 3: Guided reflectometry experimental setups : (a) incorporating the 100 $\mathrm{GHz}$ SynView unit (1) with the waveguide support (2) and the scanned test target (3); (b) using compact Si-Radar $122 \mathrm{GHz}$ chip (1), held beside the cardboard centering ring of the waveguide support (2).

\section{RESUlts}

In addition to the experimental setup implementation, 3D full-wave electromagnetic simulations are performed to investigate and further characterize the propagation properties of radar signals in guided terahertz reflectometry systems. Depending on the employed radar unit, two models in association with horn antenna and patch antennas respectively are constructed. Both are simulated using the 3D full-wave simulation suite, CST microwave studio, with a time-domain solver applying finite integration technique while the profiles of the electric fields are recorded for further performances evaluations. It should be pointed out that no material losses (neither in dielectric materials nor in metals) are considered in those simulations.

\section{A. $100 \mathrm{GHz}$ SynView transceiver reflectometry unit}

As shown in Fig. 4a, the simulation model based on the 100 $\mathrm{GHz}$ SynView transceiver reflectometry unit is composed of a typical W-Band conical horn antenna, a $30 \mathrm{~cm}$ long plastic hollow-core waveguide and a $5 \mathrm{~cm}$ long air block. The circular input face of the conical horn antenna is selected as a port for signal excitation and detection. All the E-field results presented below are obtained at the central frequency of $100 \mathrm{GHz}$.

\section{1) Propagation characterisation}

Field distribution simulation results, displayed in Fig. 4b and $4 \mathrm{c}$, provide substantial information concerning the propagation behaviors of guided waves in the system which are tightly related to the profile of the injection source.

Over the first part of the propagation, a stabilization of the guided signal is noticeable, while towards the end of the 30 $\mathrm{cm}$ waveguide, a steady field distribution is retained which corroborates the guidance capacity of the waveguide.

However, the field distribution along the waveguide reveals that not only air-core modes, but also cladding modes are excited, resulting in the significant field confinement in the two sides of the cladding in the x-direction. Both the the antiresonant reflection and total internal reflection are exploited as guiding mechanisms. The low effective refractive index, induced by the thin cladding pipe geometry, still ensures a low attenuation and prevents any significant back-reflection from the output waveguide-air interface to restrain the parasitic standing waves formation along the waveguide. Nevertheless, with this guide geometry, the field distributions, in Fig. 4d, also display propagating modes around the cladding. The external field spillage, induced by the low confinement of this mode, enables an additional beneficial sensing capability along the waveguide. Indeed, combined with the high sensitivity and the phase information natively embedded with the FMCW sensing method, this external field distribution allows for reflective sensing and differentiation of inserted perturbations in the vicinity of the waveguide.

\section{2) Coupling-in issue}

As mentioned previously, the coupling is achieved directly by inserting the waveguide into the conical horn antenna of the radar unit to get a proper centering and field collection. To characterize the coupling efficiency of this assembly, due to the non-controlled multi-mode propagation, the electromagnetic power is evaluated at different positions along the waveguide, from integration of the Poynting vector over the surface of cross-sections. Fig. 5 depicts simulated enclosed power as a function of the propagation distance. The power features over the first $30 \mathrm{~mm}$ (Phase I), correspond to the excitation signal confined in the metallic horn antenna. The gradual decrease (Phase II) is induced by the radiation loss of noncoupled waves. The stable guided propagation then takes place along the waveguide (Phase III) where no significant power losses induced by material absorption or radiation loss caused by non-guided waves are witnessed. The coupling 
(a)

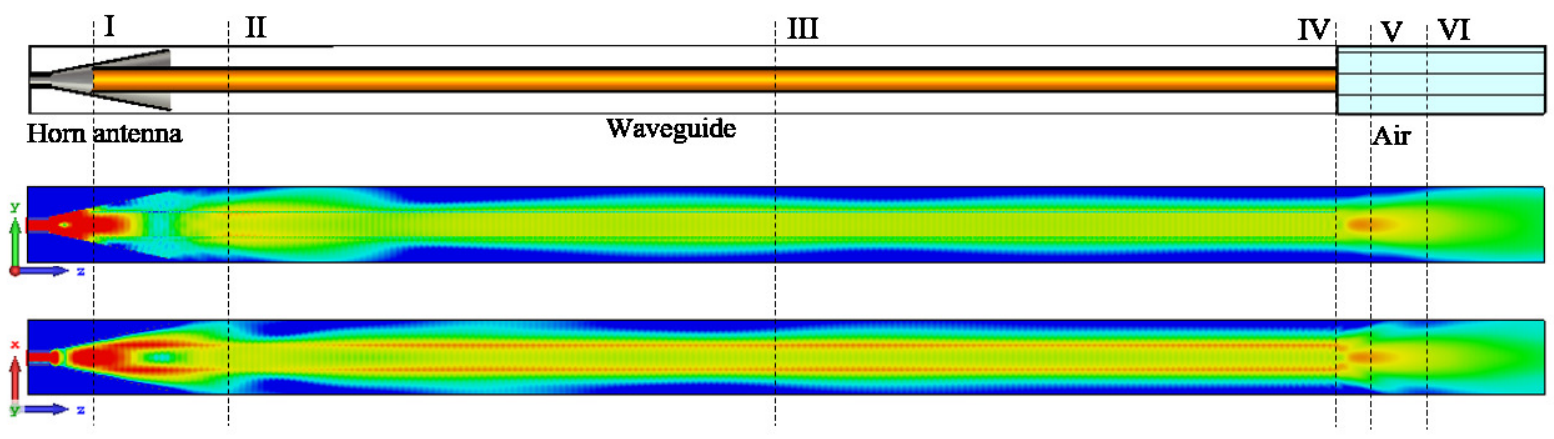

(d)
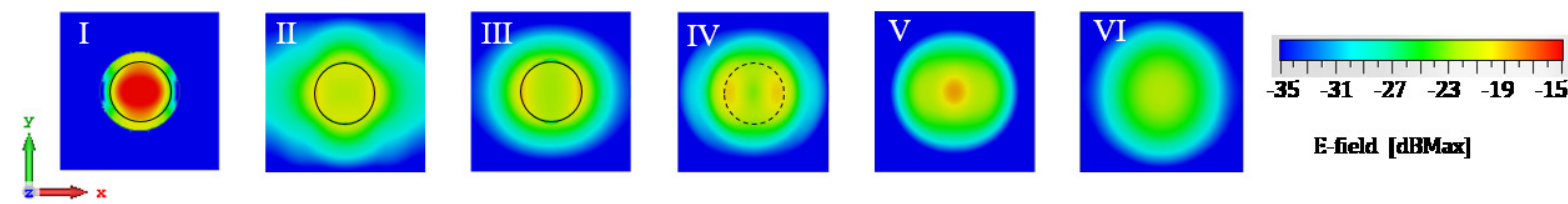

Fig. 4: (a) Diagram of the guided reflectometry radar setup using the $100 \mathrm{GHz}$ SynView unit, simulated electric field magnitude profile along (b) the z-y plane and (c) the $\mathrm{z}-\mathrm{x}$ plane, (d) $\mathrm{xy}$ cut of the electric field magnitude profile at respectively (I) $\mathrm{z}=0 \mathrm{~mm}$, (II) $\mathrm{z}=30 \mathrm{~mm}$, (III) $\mathrm{z}=190 \mathrm{~mm}$, (IV) $\mathrm{z}=300$ $\mathrm{mm}$ and (V) $5 \mathrm{~mm}$, (VI) $20 \mathrm{~mm}$ from the waveguide's output.

efficiency, dictated by Eq. 4, and hereby directly deduced from the simulation steady state, then approaches $70 \%$. Once the radar signal propagates out of the waveguide (Phase IV), the enclosed power reduction occurs due to the beam expansion in free space.

$$
\eta=\frac{P_{\text {Guided }}}{P_{\text {Excitation }}}
$$

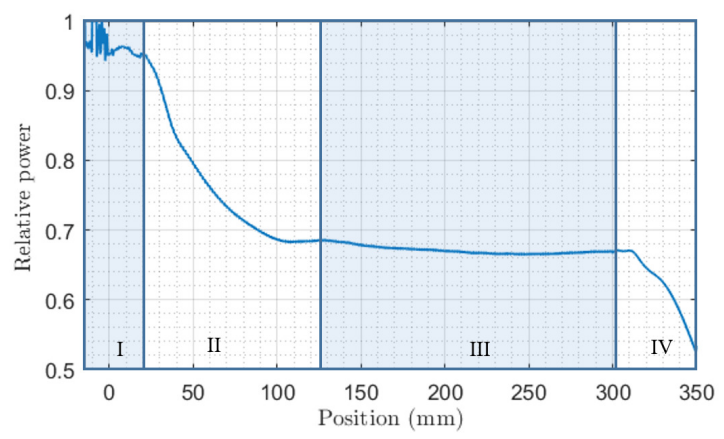

Fig. 5: Simulation of the enclosed power as a function of the propagation distance revealing the coupling ratio when reaching guided steady-state, for the $100 \mathrm{GHz}$ SynView guided module.

\section{3) Dynamic range}

For such reflectometry sensing tools, the measurement dynamic range of the unit is one of the main performance indicators for sensing purposes. The $100 \mathrm{GHz}$ SynView FMCW Radar unit itself offers a maximum $50 \mathrm{~dB}$ dynamic range when used in standard quasi-optical imaging configuration. Nevertheless, the implementation of the waveguide will ineluctably induce a limitation on this dynamic range. Indeed,impacting the signal as a constant background noise, the unavoidable signal reflection from the open probing end of the waveguide will be the main limiting factor for the dynamic range together with the power loss induced by the imperfect waveguideantenna coupling. Appropriate simulations have been conducted to asses this impact via the comparison of the reflected power for two extreme cases, with first the simulation of the open-end waveguide as a background signal characterisation and secondly, the implementation of a perfect reflector for the assessment of the optimum achievable signal level. A 28.5 dB amplitude reflection coefficient from the open-end back-reflection has been deducted from the comparison of the reflected field amplitude with the simulation input excitation. Similarly, inserting a perfect reflector at the end of the guide, a $-1.5 \mathrm{~dB}$ return signal coefficient has been deducted from those simulations, mainly impacted by the imperfect coupling from the horn antenna to the waveguide. A total maximum achievable dynamic range of $27 \mathrm{~dB}$, dictated by the ratio between the previous extreme reflection coefficients can then be expected.

\section{4) Optical resolution and imaging capabilities}

The free space propagation behaviors after the waveguide is of interest to characterize the sensing and imaging capabilities of the entire system. Indeed, the propagation properties of radar waves leaving the waveguide determine the optimum sensing distance as well as the lateral resolution at the probing point. While the maximum sensing distance is related to the back-coupling efficiency, the resolution capabilities is established by the beam profile, the latter being especially important for imaging purposes. Fig. $4 d$ depicts the simulated electric field distribution in the $x y$-plane at different positions. It can be observed that, although the back-coupling efficiency is optimized in the vicinity of the waveguide's output, (Fig. 4d (IV), the beam profiles display 2 main amplitude lobes confined in the opposite sides of waveguide's cladding. This inhomogeneity directly leads to ghost imaging artifacts causing a duplication of the object when using the system in such a close configuration.

The impact of this optical profile heterogeneity is noticeable on the image of a metal-on-PCB USAF resolution test chart, performed by classical $x-y$ raster-scanning method, resulting from collected radar data in the waveguide output plane, where some vertical elements are duplicated (see Fig. 6b). Neverthe- 
less, after propagation, the beam profile is then smoothed due to the spreading of the two electric fields lobes (see Fig. 4d $(\mathrm{V})$ ). It reach an optimum imaging resolution of $4.5 \mathrm{~mm}$ at Full Width Half Maximum thanks to a quite homogeneous profile at an optimum working distance of $5 \mathrm{~mm}$. Working at larger distances, the back-coupling efficiency along with the

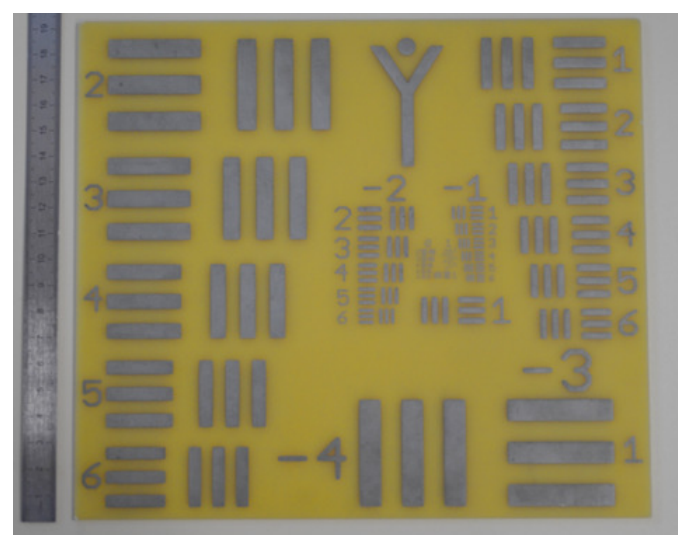

(a)

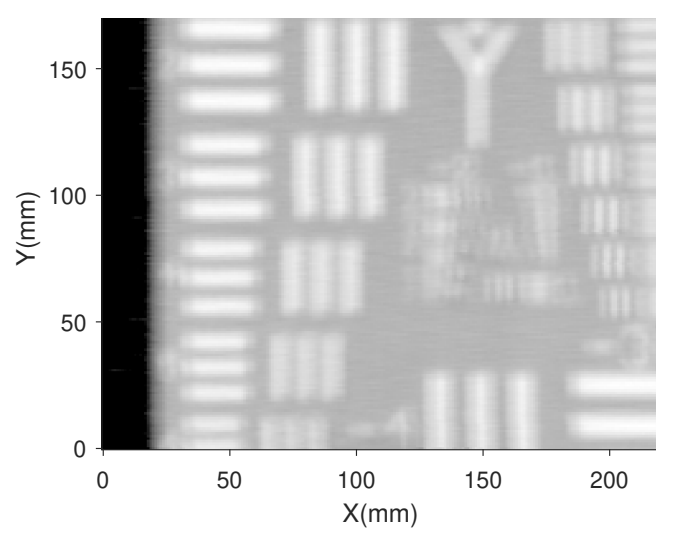

(b)

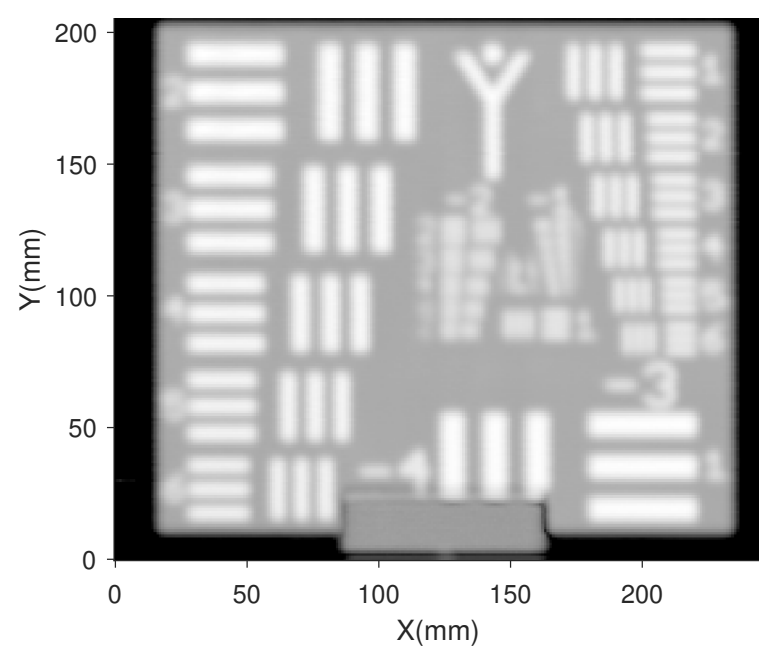

(c)

Fig. 6: (a) Photograph of test chart. (b) Raster scan acquired image, with $1 \mathrm{~mm}$ step size, using the guided $100 \mathrm{GHz}$ Synwiew probing system in lensless geometry at $1 \mathrm{~mm}$ imaging distance. (c) Raster scan acquired image using the regular far-field $100 \mathrm{GHz}$ SynView unit at $\mathrm{NA}=0.5$ for reference, with $1 \mathrm{~mm}$ step size. resolution will decrease due to the free-space beam spreading, leading to a trade-off between the back-coupling efficiency and lateral resolution if larger working distances are required. For comparative purposes, the far field image obtained with the classical quasi optical implementation of the $100 \mathrm{GHz}$ SynView unit is displayed in Fig. 6c.

5) Optical resolution improvement: solid immersion lens implementation

To address the limitations of the imaging resolution linked to the waveguide's diameter while avoiding heavy configuration with optical components, an end-of-waveguide solid immersion lens has been selected as an adequate solution. Thanks to their specific geometries and materials, typically hemispherical, hypo or hyper-hemispherical or bullet dielectric lenses designs, solid immersion lenses allow high Numerical Aperture (NA) focusing for high resolution imaging and short working distances.

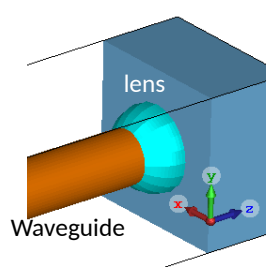

(I)

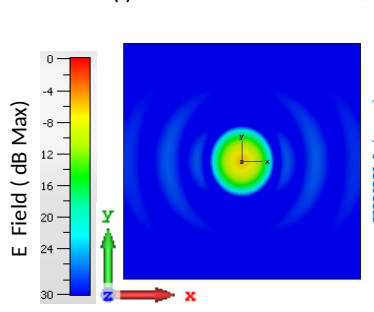

(IV)

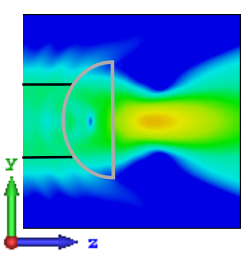

(II)

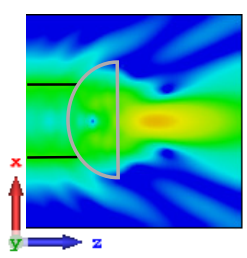

(III)

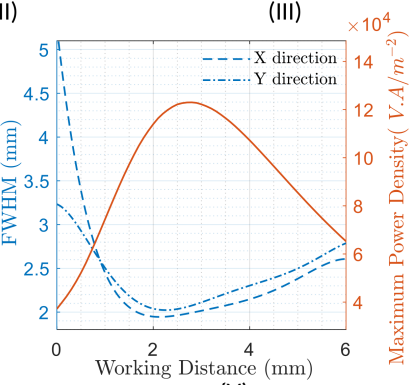

(V) (a)

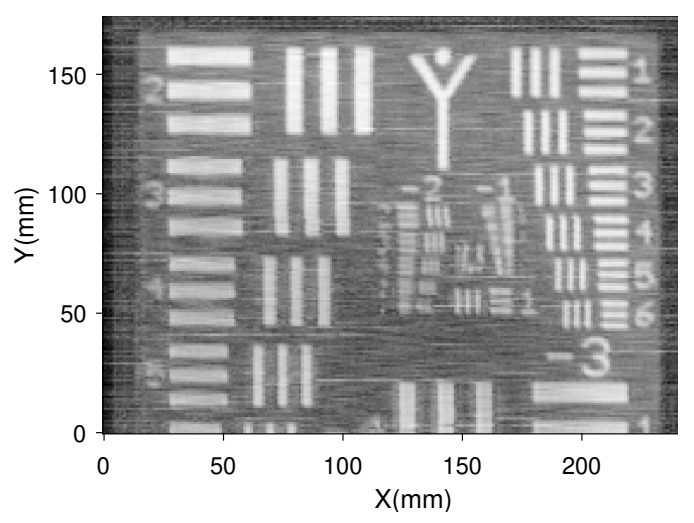

(b)

Fig. 7: (a) Electromagnetic simulations with solid immersion HDPE hemispherical lens : (I) implementation diagram of the guide termination, electric field magnitude profile (II) in z-y plan, (III) $\mathrm{z}-\mathrm{x}$ plan and (IV) $\mathrm{x}-\mathrm{y}$ cut in the best imaging plan, (V) evolution of the beam diameter and maximum power density with respect to the working distance. (b) Raster scan acquired image, with $1 \mathrm{~mm}$ step size, using the guided Synwiew sensing unit with hemispherical HDPE termination lens at $2.5 \mathrm{~mm}$ imaging distance.

In the experiment, a $9 \mathrm{~mm}$ diameter hemispherical High Density Polyethylene (HDPE) lens is inserted at the output of 
(a)

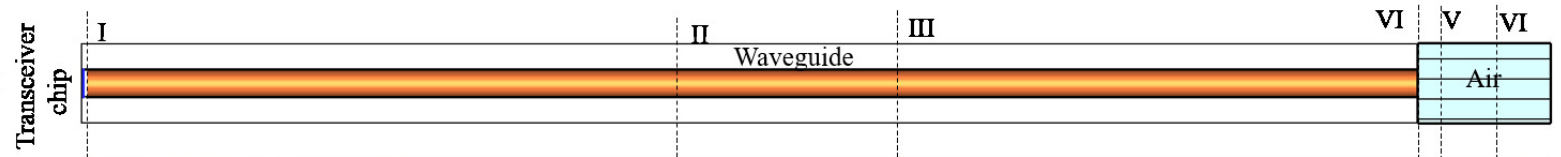

(b)

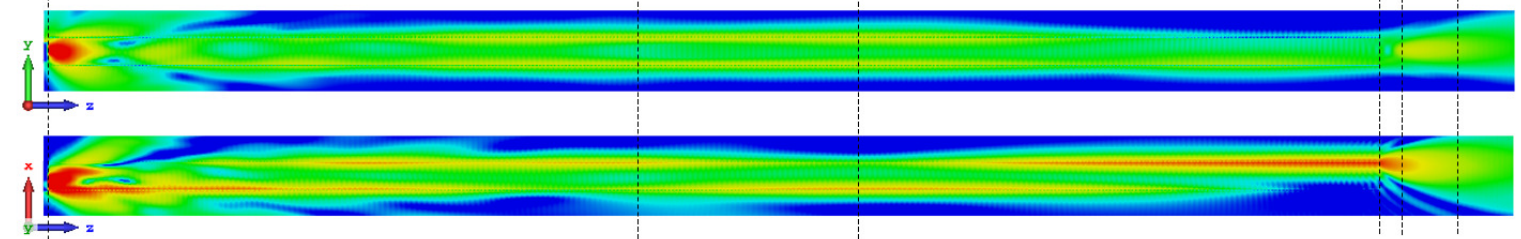

(d)
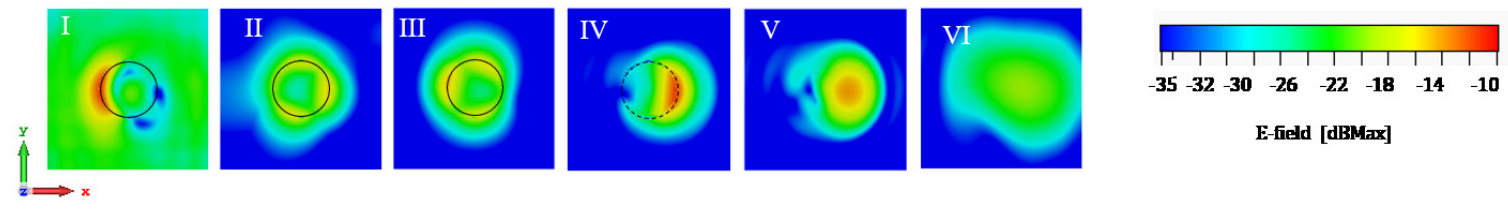

Fig. 8: (a) Diagram of the guided reflectometry radar setup using the $122 \mathrm{GHz}$ Silicon radar chip, simulated electric field magnitude profile along (b) the $\mathrm{z}-\mathrm{y}$ plan and (c) the $\mathrm{z}$-x plane, (d) $\mathrm{x}-\mathrm{y}$ cut of the electric field magnitude profile at respectively (I) $\mathrm{z}=0 \mathrm{~mm}$, (II) $\mathrm{z}=140 \mathrm{~mm}$, (III) $\mathrm{z}=180 \mathrm{~mm}$, (IV) $\mathrm{z}=300$ $\mathrm{mm}$ and (V) $3 \mathrm{~mm}$, (VI) $20 \mathrm{~mm}$ from the output of the waveguide.

the waveguide (see Fig. 7a (I)). Its relatively low refractive index induces a moderate unwanted signal return from the interfaces while contact-less sensing is still ensured by the back working distance linked to its diameter. Indeed, simulating this lens geometry at the output of the waveguide unit (see Fig. 7a (II) (III)), a beam optimum spot size of $2 \mathrm{~mm}$ at Full Width at Half Maximum in $\mathrm{X}$ and $\mathrm{Y}$ directions is obtained at a frontal working distance of $2.5 \mathrm{~mm}$, along with the optimum power density observed around this point (see Fig. 7a (IV) (V)) while residual low amplitude side lobes at $-25 \mathrm{~dB}$ are induced by the waveguide spillage. This simulated profile corroborates the resolution of the raster scan acquired image of a test chart where we found a $2 \mathrm{~mm}$ resolution (group -2, element 1) (see Fig. $7 b$ ) is achieved around the expected simulated optimum working distance of $2 \mathrm{~mm}$ and depicts a drastic improvement compared to the guided lens-less FMCW implementation or a classic FMCW far-field imaging setup. Working distances beyond or closer from this optimum point obviously degrades the achievable resolution and are drastically impacting the back coupling efficiency.

\section{B. $122 \mathrm{GHz}$ Silicon radar chip reflectometry unit}

Focusing on the low-cost alternative unit, as illustrated in Fig. 8a, the simulation setup remains similar to the previous model with a simple substitution of the transceiver module, which now consists of two $2 * 2$ patch antennas as TX and RX providing a wide emission pattern. Limited by the simulation voxel's size definition and so the calculation capacity, the transceiver chip is replaced by its equivalent source. All Efield results presented in the following part are obtained at the operational bandwidth central frequency of $122 \mathrm{GHz}$.

Compared to the $100 \mathrm{GHz}$ SynView head, Siliconradar chip displays a different front-end architecture: patch antennas lead to a much broader emission pattern. Significant modifications of the electric field distribution along the waveguide were expected and is depicted in Fig. 8. Contrary to the symmetrical integration with a horn antenna, obvious signal reflections on the waveguide's cladding can be observed through the oscillating behavior during the propagation. This can be explained by the decentering of the transmitter with respect to the waveguide, the excitation conditions not being symmetrical anymore. It is worth noting that the non-negligible field spillage locating at the waveguide's periphery grants the peripheral sensing capability.

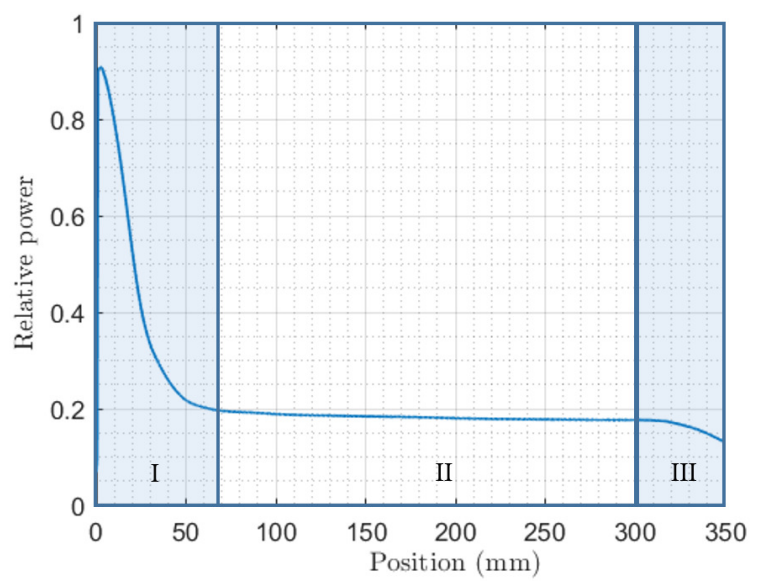

Fig. 9: Simulation of the enclosed power as a function of propagation distance revealing the coupling ratio for the $122 \mathrm{GHz}$ Si-Based radar chip guided module.

Owing to the wide emission pattern of the patch antenna design, a considerable power fraction is radiated into free space rather than coupled into the waveguide. Fig. 9 Phase I depicts this power loss while phase II indicates that the radar signal reaches guided power stabilization after a $50 \mathrm{~mm}$ propagation. As a direct consequence, the coupling efficiency is reduced to $18 \%$ and mainly impacted by the near-isotropic emission profile of the patch antennas, compared to the previously achieved $70 \%$ with the directional emission of the horn antenna of the former setup. Moreover, based on remaining field energy after open-end reflection and perfect mirror reflection, the estimated full dynamic range can reach up to 
27.6 dB. Although the dynamic ranges of this reflectometry system are similar in comparison to the SynView $100 \mathrm{GHz}$ architecture a much larger coupling loss is witnessed in the system using the $122 \mathrm{GHz}$ integrated radar chip.

An image of the test chart obtained in this reflectometry configuration is provided in Fig. 10a. The achieved resolution of $2.8 \mathrm{~mm}$ (group -3 element 4), directly at the output of the waveguide, corroborates the simulated Full Width at Half Maximum output's beam diameter, thus reaching a better resolution level than the previously described reference system. The atypical coupling-in geometry gives rise to an asymmetric field distribution at the output of the waveguide, where the highest electric field is confined at one side of the core's cross section (Fig. 8c and d (IV)). Even though an irregular beam pattern is emitted, the single lobe profile output field distribution ensures no significant ghost artefacts but the asymmetry of the emission and the back coupling induce a shadowing effect, noticeable on the image. In spite of it, within the longitudinal resolution imposed by the radar bandwidth the sample support on the backside can be seen in the center bottom part of the image, demonstrating the in-depth sensing capability of the system.

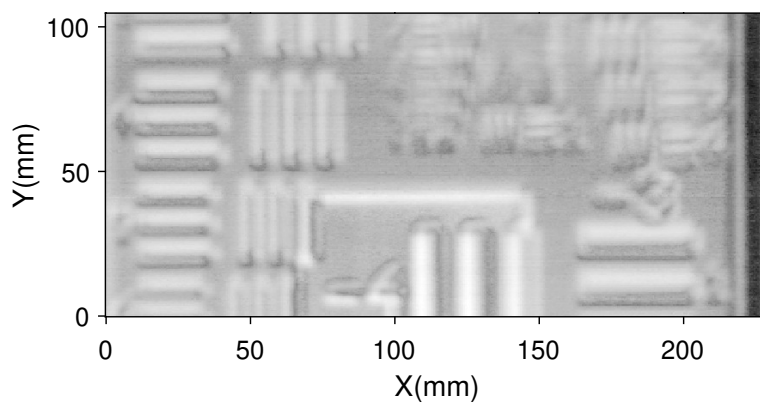

(a)

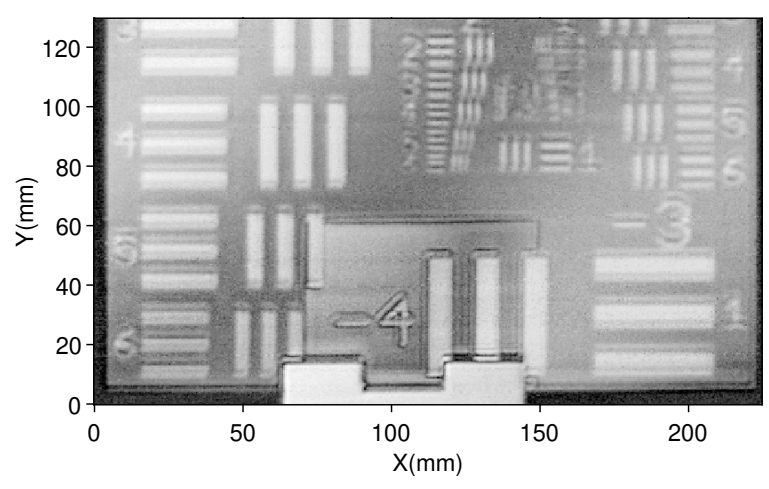

(b)

Fig. 10: Raster scan images, with $500 \mu \mathrm{m}$ step size, of a test chart using the guided $122 \mathrm{GHz}$ Si-radar probing system in two configurations: (a) in lensless geometry with an imaging distance of $1 \mathrm{~mm}$ and (b) in association with a hemispherical PE termination lens with an imaging distance of $3 \mathrm{~mm}$.

Similarly to our reference setup, for the sake of imaging resolution improvement, a $9 \mathrm{~mm}$ diameter hemispherical HDPE termination lens has been inserted to counteract the free space propagation beam divergence and reach a better resolved focus point. Fig. 10b displays the raster scan acquired image of the test chart in this configuration where a $1.4 \mathrm{~mm}$ resolution is achieved (group -2 element 4 ) in accordance with the conducted beam profile simulations, better than the resolution obtained with the reference $100 \mathrm{GHz}$ guided SynView unit. Nevertheless ghost artefacts are still noticeable and induced by beam profile inhomogeneities leading to an improper beam focusing with non-negligible side lobes, witnessed as well on those simulations.

\section{CONCLUSIONS}

In this work, guided terahertz FMCW reflectometry probing systems have been demonstrated as a low-cost solution where a polymer pipe waveguide is used to ensure the single coupling channel to reach a compact and simplified guided probing unit. Two architectures have been investigated to implement a high performances unit as well as a low-cost solution. With the help of the 3D full-wave electromagnetic simulations, the propagation behaviors in the guided systems have been investigated to corroborate implementation results. In particular, a quantitative analysis focused on waveguide coupling efficiency, expected sensing dynamic range and optical resolution have been performed. Finally, imaging capabilities of those guided systems are demonstrated by the raster-scanning method on test charts, which supplement and validate the simulations as well as the waveguide propagation induced artefacts. Intrinsically limited by the waveguide's dimension, further improvements on the imaging resolution have been investigated through the implementation of solid immersion lenses while ongoing work are directed towards the design and selection of those lenses according to their intended use as well as the selection of an improved waveguide dimension for better coupling performances with both systems.

\section{ACKNOWLEDGMENT}

This work was supported in part by the French Ministry of National Education, Research and Technology. Authors acknowledge Dr. Damien Bigourd and Dr. Quentin Cassar for their interesting discussions, advises upon terahertz waveguides and their additional checks of this article.

\section{REFERENCES}

[1] P. Hillger, J. Grzyb, R. Jain, and U. R. Pfeiffer, "Terahertz imaging and sensing applications with silicon-based technologies," IEEE Transactions on Terahertz Science and Technology, vol. 9, no. 1, pp. 1-19, 2018.

[2] S. Dhillon, M. Vitiello, E. Linfield, A. Davies, M. C. Hoffmann, J. Booske, C. Paoloni, M. Gensch, P. Weightman, G. Williams, et al., "The 2017 terahertz science and technology roadmap," Journal of Physics D: Applied Physics, vol. 50, no. 4, p. 043001, 2017.

[3] J.-B. Perraud, J.-P. Guillet, O. Redon, M. Hamdi, F. Simoens, and P. Mounaix, "Shape-from-focus for real-time terahertz 3d imaging," Optics letters, vol. 44, no. 3, pp. 483-486, 2019.

[4] J. B. Perraud, A. F. Obaton, J. Bou-Sleiman, B. Recur, H. Balacey, F. Darracq, J.-P. Guillet, and P. Mounaix, "Terahertz imaging and tomography as efficient instruments for testing polymer additive manufacturing objects," Applied optics, vol. 55, no. 13, pp. 3462-3467, 2016.

[5] T. M. Wong, M. Kahl, P. H. Bolívar, and A. Kolb, "Frequency modulated continuous wave (fmcw) thz image $3 \mathrm{~d}$ superresolution," arXiv preprint arXiv:1802.05457, 2018.

[6] F. Friederich, K. May, B. Baccouche, C. Matheis, M. Bauer, J. Jonuscheit, M. Moor, D. Denman, J. Bramble, and N. Savage, "Terahertz radome inspection," in Photonics, vol. 5, no. 1. Multidisciplinary Digital Publishing Institute, 2018, p. 1. 
[7] E. Cristofani, F. Friederich, S. Wohnsiedler, C. Matheis, J. Jonuscheit, M. Vandewal, and R. Beigang, "Nondestructive testing potential evaluation of a terahertz frequency-modulated continuous-wave imager for composite materials inspection," Optical Engineering, vol. 53, no. 3, p. 031211, 2014.

[8] J.-P. Guillet, M. Roux, K. Wang, X. Ma, F. Fauquet, H. Balacey, B. Recur, F. Darracq, and P. Mounaix, "Art painting diagnostic before restoration with terahertz and millimeter waves," Journal of Infrared, Millimeter, and Terahertz Waves, vol. 38, no. 4, pp. 369-379, 2017.

[9] G. Humbert, Optical Fibers in Terahertz Domain. Singapore: Springer Singapore, 2019, pp. 1-49.

[10] M. Pan, Q. Cassar, F. Fauquet, G. Humbert, P. Mounaix, and J.-P. Guillet, "Guided terahertz pulse reflectometry with double photoconductive antenna," Appl. Opt., vol. 59, no. 6, pp. 1641-1647, Feb 2020.

[11] Y. Gao and R. Zoughi, "Millimeter wave reflectometry and imaging for noninvasive diagnosis of skin burn injuries," IEEE Transactions on Instrumentation and Measurement, vol. 66, no. 1, pp. 77-84, 2017.

[12] B. You and J.-Y. Lu, "Remote and in situ sensing products in chemical reaction using a flexible terahertz pipe waveguide," Optics express, vol. 24, no. 16, pp. 18013-18 023, 2016.

[13] J.-P. Guillet, L. Chusseau, R. Adam, T. Grosjean, A. Penarier, F. Baida, and D. Charraut, "Continuous-wave scanning terahertz near-field microscope," Microwave and Optical Technology Letters, vol. 53, no. 3, pp. 580-582, 2011.

[14] P. Doradla, K. Alavi, C. S. Joseph, and R. H. Giles, "Flexible waveguide enabled single-channel terahertz endoscopic system," in Terahertz, $R F$, Millimeter, and Submillimeter-Wave Technology and Applications VIII, vol. 9362. International Society for Optics and Photonics, 2015, p. 93620D.

[15] J. Hasch, E. Topak, R. Schnabel, T. Zwick, R. Weigel, and C. Waldschmidt, "Millimeter-wave technology for automotive radar sensors in the $77 \mathrm{ghz}$ frequency band," IEEE Transactions on Microwave Theory and Techniques, vol. 60, no. 3, pp. 845-860, 2012.

[16] M. Pauli, B. Göttel, S. Scherr, A. Bhutani, S. Ayhan, W. Winkler, and T. Zwick, "Miniaturized millimeter-wave radar sensor for high-accuracy applications," IEEE Transactions on Microwave Theory and Techniques, vol. 65, no. 5, pp. 1707-1715, 2017.

[17] K. Schmalz, W. Winkler, J. Borngräber, W. Debski, B. Heinemann, and J. Scheytt, "122 ghz ism-band transceiver concept and silicon ics for low-cost receiver in sige bicmos," in 2010 IEEE MTT-S International Microwave Symposium. IEEE, 2010, pp. 1332-1335.

[18] K. Schmalz, J. Borngräber, B. Heinemann, H. Rücker, and J. Scheytt, "A 245 ghz transmitter in sige technology," in 2012 IEEE Radio Frequency Integrated Circuits Symposium. IEEE, 2012, pp. 195-198.

[19] A. Maestrini, J. Ward, J. Gill, H. Javadi, E. Schlecht, G. Chattopadhyay, F. Maiwald, N. R. Erickson, and I. Mehdi, "A 1.7-1.9 thz local oscillator source," IEEE Microwave and Wireless Components Letters, vol. 14, no. 6, pp. 253-255, 2004.

[20] M. Abbasi, S. E. Gunnarsson, N. Wadefalk, R. Kozhuharov, J. Svedin, S. Cherednichenko, I. Angelov, I. Kallfass, A. Leuther, and H. Zirath, "Single-chip 220-ghz active heterodyne receiver and transmitter mmics with on-chip integrated antenna," IEEE Transactions on Microwave Theory and Techniques, vol. 59, no. 2, pp. 466-478, 2010.

[21] K. B. Cooper, R. J. Dengler, N. Llombart, T. Bryllert, G. Chattopadhyay, E. Schlecht, J. Gill, C. Lee, A. Skalare, I. Mehdi, et al., "Penetrating 3-d imaging at 4-and $25-\mathrm{m}$ range using a submillimeter-wave radar," IEEE Transactions on Microwave Theory and Techniques, vol. 56, no. 12, pp. 2771-2778, 2008

[22] C. Am Weg, W. von Spiegel, R. Henneberger, R. Zimmermann, T. Loeffler, and H. G. Roskos, "Fast active thz cameras with ranging capabilities," Journal of Infrared, Millimeter, and Terahertz Waves, vol. 30, no. 12, pp. 1281-1296, 2009.

[23] C.-H. Lai, B. You, J.-Y. Lu, T.-A. Liu, J.-L. Peng, C.-K. Sun, and H.-C Chang, "Modal characteristics of antiresonant reflecting pipe waveguides for terahertz waveguiding," Optics express, vol. 18, no. 1, pp. 309-322, 2010.

[24] E. Nguema, D. Férachou, G. Humbert, J.-L. Auguste, and J.-M. Blondy, "Broadband terahertz transmission within the air channel of thin-wall pipe," Optics letters, vol. 36, no. 10, pp. 1782-1784, 2011.

[25] B. Bowden, J. A. Harrington, and O. Mitrofanov, "Silver/polystyrenecoated hollow glass waveguides for the transmission of terahertz radiation," Optics letters, vol. 32, no. 20, pp. 2945-2947, 2007.

[26] Y. Geng, X. Tan, P. Wang, and J. Yao, "Transmission loss and dispersion in plastic terahertz photonic band-gap fibers," Applied Physics B, vol. 91, no. 2, pp. 333-336, 2008.

[27] I. K. Yakasai, P. E. Abas, S. Ali, and F. Begum, "Modelling and simulation of a porous core photonic crystal fibre for terahertz wave propagation," Optical and Quantum Electronics, vol. 51, no. 4, p. 122, 2019.

[28] M. A. Habib, M. S. Reza, L. F. Abdulrazak, and M. S. Anower, "Extremely high birefringent and low loss microstructure optical waveguide: Design and analysis," Optics Communications, vol. 446, pp. 93-99, 2019.

[29] C.-H. Lai, Y.-C. Hsueh, H.-W. Chen, Y.-j. Huang, H.-c. Chang, and C.K. Sun, "Low-index terahertz pipe waveguides," Optics letters, vol. 34, no. 21, pp. 3457-3459, 2009.

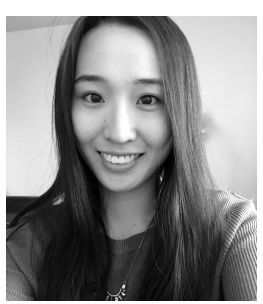

Mingming Pan was born in Jiangsu, China, in 1992. She received the B.Sc. degree in electronics from the University of Suzhou, Suzhou, China, in 2013, and the M.Sc. degree in electronics from Bordeaux University, Bordeaux, France, where she is currently working toward the Ph.D. degree in electronics, entitled "Reflectometry in time-domain for guided terahertz waves." She was involved at IMS Laboratory (UMR CNRS 5218) on emission and detection of $\mathrm{THz}$ waves techniques.

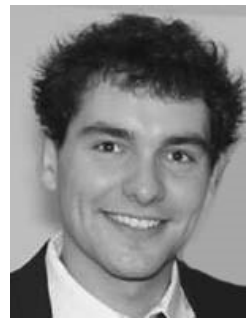

Adrien Chopard was born in France, in 1993. He received the Diplome d'Ingénieur in optics and photonics from the Institut d'Optique Graduate School (IOGS-Saclay), Palaiseau, France, in 2016 and the M.Sc. degree in engineering specialized in nanotechnology from the Royal Institute of Technology of Sweden, (KTH), Stockholm, Sweden. He is currently working toward the industrial Ph.D. degree in physics on applicative developments using terahertz technologies, with the firm Lytid based in Paris and the IMS Laboratory (UMR CNRS 5218), University of Bordeaux, Talence, France.

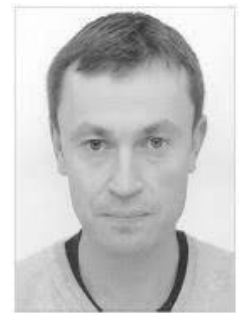

Frederic Fauquet was born in Pessac, France, in 1977. He received the Diplome Universitaire en Technologie in electronics from Bordeaux University, Bordeaux, France, in 1998. He worked for 14 years with the Observatoire de Physique du Globe (OPGS UMS 833), Clermont-Ferrand, France, where he contributed to the development of autonomous multisensor station. In 2014, he moved to Bordeaux to join the laser and terahertz team at IMS Laboratory as a Research Engineer. 


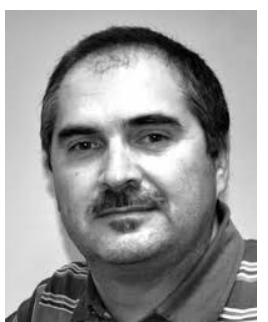

Patrick Mounaix received the Engineering degree in material science from the École Universitaire D'Ingénieur de Lille (EUDIL), Villeneuve-d'Ascq, France, in 1988, and the Ph.D. degree in quantum devices from the University of Lille, Lille, France, in 1992. He joined CNRS and the High Frequency Department, Institut d'Électronique et de Microélectronique du Nord (IEMN UMR 8520). In 2002, he moved to Bordeaux where he developed terahertz spectroscopy and imaging activities. He is currently a Senior Researcher with IMS Laboratory (UMR CNRS 5218), Bordeaux University, Bordeaux, France. He has coauthored more than 210 papers. His current research interests include equilibrium and time-resolved terahertz spectroscopy techniques for solid and liquid materials and 3-D terahertz imaging applications, chiefly 3-D computed tomography for art science and NDE industrial applications. He also works on all dielectric metamaterials and their applications at microwave and terahertz frequency range. He has coauthored more than 210 papers.

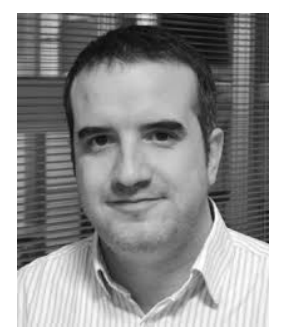

Jean-Paul Guillet was born in Lyon, France, in 1984. He received the M.Sc. and Ph.D. degrees in terahertz imaging from the IES Lab, Montpellier, France, in 2007 and 2010, respectively. The topic of this first research work was terahertz near-field microscopy using electronics-based components. During his postdoctoral studies, until 2013, his research focused on imaging and tomography, using femtosecond pulses, frequency-modulated continuouswave approach, and a terahertz camera. In 2013, he was an Associate Professor with the IMS (UMR CNRS 5218), University of Bordeaux, where he is currently focusing on very large-scale integration circuits fault isolation laser techniques and terahertz imaging. 